Pacific Journal of Mathematics

A PROPERTY OF SOME FOURIER-STIELTJES TRANSFORMS 


\title{
A PROPERTY OF SOME FOURIER-STIELTJES TRANSFORMS
}

\author{
HiRoshi YamaguchI
}

\begin{abstract}
Helson and Lowdenslager extended the classical F. and M. Riesz theorem as follows:

Let $G$ be a compact abelian group with the ordered dual $\hat{G}$. Let $\mu$ be a bounded regular measure on $G$ which is of analytic type. Then $\mu_{a}$ and $\mu_{s}$ are of analytic type.

Doss extended this theorem for a LCA group with the algebraically ordered dual. On the other hand, deLeeuw and Glicksberg obtained an analogous result for a compact abelian group $G$ such that there exists a nontrivial homomorphism from $\hat{G}$ into $R$. In this paper, we prove that the theorem of Helson and Lowdenslager is satisfied for a LCA group with partially ordered dual.
\end{abstract}

1. Introduction. Let $G$ be a LCA group with the dual group $\hat{G}$. We denote by $m_{G}$ the Haar measure on $G$. Let $M(G)$ be the Banach algebra of bounded regular measures on $G$ under convolution multiplication and the total variation norm. $M_{s}(G)$ and $L^{1}(G)$ denote the closed subspace of $M(G)$ consisting of measures which are singular with respect to $m_{G}$ and the closed ideal of $M(G)$ consisting of measures which are absolutely continuous with respect to $m_{G}$ respectively. We denote by $\operatorname{Trig}(G)$ the set of all trigionometric polynomials on $G$. For a subset $E$ of $\hat{G}, M_{E}(G)$ denotes the space of measures in $M(G)$ whose Fourier-Stieltjes transforms vanish off $E . E^{-}$(or $\bar{E}$ ) means the closure of $E$. Let $M^{+}(G)$ be the subset of $M(G)$ consisting of positive measures. For $\mu \in M(G), \mu_{a}$ and $\mu_{s}$ denote the absolutely continuous part and the singular part of $\mu$ respectively. For a subgroup $H$ of $G, H^{\perp}$ means the annihilator of $H$.

Helson and Lowdenslager extended the classical F. and M. Riesz theorem as follows:

THEOREM A ( $c f$. [8], 8.2.3. Theorem). Let $G$ be a compact abelian group with ordered dual, i.e., there exists a semigroup $P$ in $\hat{G}$ such that (i) $P \cup(-P)=\hat{G}$ and (ii) $P \cap(-P)=\{0\}$. Let $\mu$ be a measure in $M(G)$ such that $\hat{\mu}(\gamma)=0$ for $\gamma<0$. Then

(I) $\hat{\mu}_{a}(\gamma)=\hat{\mu}_{s}(\gamma)=0$ for $\gamma<0$

(II) $\hat{\mu}_{s}(0)=0$.

In [3] and [4], Doss extended Theorem A for a LCA group. 
THeOREM B ([4], Lemma 1). Let $G$ be a LCA group such that $\hat{G}$ is algebraically ordered, i.e., there exists a semigroup $P$ in $\hat{G}$ such that (i) $P \cup(-P)=\hat{G}$ and (ii) $P \cap(-P)=\{0\}$ (we do not assume the closedness of $P)$. Let $\mu$ be a measure in $M(G)$ such that $\hat{\mu}(\gamma)=0$ for $\gamma<0$. Then

(I) $\hat{\mu}_{a}(\gamma)=\hat{\mu}_{s}(\gamma)=0$ for $\gamma<0$;

(II) $\hat{\mu}_{s}(0)=0$.

REMARK 1.1. In Theorem B, when $G$ is noncompact, (II) is obtained from (I) and the fact that 0 is an accumulation point of $P^{c}$.

On the other hand, deLeeuw and Glicksberg in [2] obtained an analogous result of Theorem A for a compact abelian group $G$ such that there exists a nontrivial homomorphism $\psi$ from $\hat{G}$ into $R$ (the reals). That is,

Theorem C (cf. [2], Proposition 5.1, p. 189). Let $G$ be a compact abelian group and $\psi$ a nontrivial homomorphism from $\hat{G}$ into $R$. Put $M^{a}(G)=\{\mu \in M(G) ; \hat{\mu}(\gamma)=0$ for $\gamma \in \hat{G}$ with $\psi(\gamma)<0\}$. Let $\mu$ be $a$ measure in $M^{a}(G)$. Then $\mu_{a}$ and $\mu_{s}$ belong to $M^{a}(G)$.

REMARK 1.2. In general, however, the conclusion of Theorem $\mathrm{C}$ can not be strengthened to " $\hat{\mu}_{s}(0)=0$ ".

Our purpose in this paper is to prove that an analogous result of Theorem $\mathrm{C}$ is satisfied for a LCA group with partially ordered dual. We state the main theorem of this paper.

MAIN TheOREM. Let $G$ be a LCA group and $P$ a closed semigroup in $\hat{G}$ such that $P \cup(-P)=\hat{G}$. Let $\mu$ be a measure in $M_{P c}(G)$. Then $\mu_{a}$ and $\mu_{s}$ belong to $M_{P^{c}}(G)$.

COROLlaRY. Let $G$ be a LCA group and $P$ a semigroup in $\hat{G}$ such that $P \cup(-P)=\hat{G}$. Then the following are satisfied:

(I) for $\mu \in M_{P}(G), \mu_{a}$ and $\mu_{s}$ belong to $M_{P}(G)$;

(II) for $\mu \in M_{P^{c}}(G), \mu_{a}$ and $\mu_{s}$ belong to $M_{P^{c}}(G)$.

Proof of Corollary. Since (II) is easily obtained from the Main Theorem, we only prove (I). We note the following:

$$
\begin{array}{cl}
\hat{\mu}=0 & \text { on }(-P \backslash P) \\
\Leftrightarrow \hat{\mu}=0 & \text { on } \gamma-P \text { for all } \gamma \in(-P) \backslash P \\
\Leftrightarrow(\gamma \mu)^{\wedge}=0 & \text { on }-P \text { for all } \gamma \in P \backslash(-P) \\
\Leftrightarrow(\gamma \mu)^{\wedge}=0 & \text { on }(-P)^{-} \text {for all } \gamma \in P \backslash(-P) .
\end{array}
$$


Hence, by the Main Theorem and the fact that $(\gamma \mu)_{a}=\gamma \mu_{a}$, we obtain the corollary.

In $\S 2$, we prove Main Theorem for a $\sigma$-compact metrizable locally compact abelian group by using the theory of disintegration. In $\$ 3$ we prove the theorem for a general locally compact abelian group by using a certain lemma which is due to Pigno and Saeki ([7], Lemma 4). The author would like to thank the referee for his valuable advice.

2. $\sigma$-compact metrizable case. In this section, we prove Main Theorem for a $\sigma$-compact metrizable locally compact abelian group. We need the theory of disintegration. The following lemma can be found in ([1], Théorème 1, p. 58).

LEMMA 2.1. Let $G$ be a $\sigma$-compact metrizable LCA group and $H$ a closed subgroup of $G$. Let $\pi$ be the natural homomorphism from $G$ onto $G / H$. Let $\mu$ be a positive measure in $M(G)$ and put $\eta=\pi(\mu)$ (continuous image under $\pi)$. Then there exists a family $\left\{\lambda_{\dot{x}}\right\}_{\dot{x} \in G / H}$ consisting of positive measures in $M(G)$ with the following properties:

(1) $\dot{x} \mapsto \lambda_{\dot{x}}(f)$ is a Borel measurable function for each bounded Borel measurable function $f$ on $G$,

(2) $\operatorname{supp}\left(\lambda_{\dot{x}}\right) \subset \pi^{-1}(\{\dot{x}\})$,

(3) $\left\|\lambda_{\dot{x}}\right\| \leq 1$,

(4) $\mu(g)=\int_{G / H} \lambda_{\dot{x}}(g) d \eta\left(_{\dot{x}}\right)$ for each bounded Borel measurable function $g$ on $G$.

Conversely, let $\left\{\lambda_{\dot{x}}^{\prime}\right\}_{\dot{x} \in G / H}$ be a family of positive measures in $M(G)$ which satisfies (1), (2) and (4). Then we have

(5) $\lambda_{\dot{x}}=\lambda_{\dot{x}}^{\prime}$ a.a. $\dot{x}(\eta)$.

Lemma 2.2. Let $G, H$ and $\pi$ be as in Lemma 2.1. Let $\mu$ be a positive measure in $M(G)$ and put $\eta=\pi(\mu)$. By (2) of Lemma 2.1, $\lambda_{\dot{x}}$ can be represented as follows:

(1) $\lambda_{\dot{x}}=\nu_{\dot{x}} * \delta_{x}$ for some $\nu_{\dot{x}} \in M^{+}(H)$ and $x \in G$ with $\pi(x)=x_{\dot{x}}$. If $\nu_{\dot{x}} \in M_{s}(H)$ a.a. $\dot{x}(\eta)$, we have $\mu \in M_{s}(G)$.

Proof. It is sufficient to prove the lemma when $\mu$ has compact support, so we can assume $\eta$ supported by $K$ compact. Suppose $\left\{f_{n}\right\} \subset$ $C_{0}(G)$ is dense. Let $\varepsilon$ be a positive real number. Then for each $n$ Lusin's theorem says $\dot{x} \mapsto \lambda_{\dot{x}}\left(f_{n}\right)$ is continuous on a compact subset $E_{n}$ of $K$ with $\eta\left(K \backslash E_{n}\right)<\varepsilon / 2^{n}$. We put $E=\bigcap_{n=1}^{\infty} E_{n}$. Then $E$ is compact, $\eta(K \backslash E)<\varepsilon$ 
and $\dot{x} \mapsto \lambda_{\dot{x}}\left(f_{n}\right)$ is continuous on $E$ for all $n$. Hence $\dot{x} \mapsto \lambda_{\dot{x}}(h)$ is continuous on $E$ for all $h \in C_{0}(G)$. By the hypothesis we may assume that $\left\|\lambda_{\dot{x}}\right\|=1$ and $\nu_{\dot{x}} \in M_{s}(H)$ for all $\dot{x} \in E$. Hence for $\dot{x} \in E$ we can choose $f=f_{\dot{x}} \in C_{c}(G)$ with $0 \leq f \leq 1,1=\left\|\lambda_{\dot{x}}\right\|<\lambda_{\dot{x}}(f)+\varepsilon$ and $\delta_{x} * m_{H}(f)<\varepsilon$ $\left(x \in \pi^{-1}(\{\dot{x}\})\right)$. Then both inequalities are held on some neighborhood of $x$ in $E$, say $N_{\dot{x}}$. Since $E$ lies in $N_{\dot{x}_{1}}, \ldots, N_{\dot{x}_{k}}$, with $f_{1}, \ldots, f_{k}$ the corresponding $f$ 's, we set $g=f_{1}$ on $\pi^{-1}\left(N_{\dot{x}_{1}}\right)$, $=f_{2}$ on $\pi^{-1}\left(N_{\dot{x}_{2}} \backslash N_{\dot{x}_{1}}\right), \ldots,=f_{k}$ on $\pi^{-1}\left(N_{\dot{x}_{k}} \backslash \cup_{j=1}^{k-1} N_{\dot{x}_{j}}\right)$ and $=0$ on $\pi^{-1}\left(E^{c}\right)$. Then $g$ is a Borel measurable function on $G$ with $0 \leq g \leq 1$ satisfying $1-\varepsilon<\lambda_{\dot{x}}(g)$ and $\delta_{x} * m_{H}(g)<\varepsilon$ for all $\dot{x} \in E(x \in \pi(\{\dot{x}\}))$. Thus $\mu(g)=\int_{G / H} \lambda_{\dot{x}}(g) d \eta(\dot{x})>1-2 \varepsilon$ and $m_{G}(g)<\varepsilon m_{G / H}(K)$. Since this holds for each $\varepsilon>0, \mu$ is necessarily singular.

LEMMA 2.3. Let $G$ be a LCA group and $H$ a closed subgroup of $G$. Let $\pi$ be the natural homomorphism from $G$ onto $G / H$. Let $\mu$ be a measure in $M^{+}(G)$. If $\pi(\mu)$ belongs to $M_{s}(G / H), \mu$ is singular with respect to the Haar measure on $G$.

Proof. Since $\pi(\mu) \in M_{s}(G / H)$, there exists a $\sigma$-compact subset $\tilde{E}$ of $G / H$ such that $\pi(\mu)\left(\tilde{E}^{c}\right)=0$ and $m_{G / H}(\tilde{E})=0$. Then $\mu$ is concentrated on $\pi^{-1}(\tilde{E})$. Therefore it is sufficient to prove that $\pi^{-1}(\tilde{E})$ is a locally null set. For a compact set $K$ in $G$, we have

$$
\begin{aligned}
m_{G}\left(K \cap \pi^{-1}(\tilde{E})\right) & =\int_{G} \chi_{K}(x) \chi_{\pi^{-1}(\tilde{E})}(x) d m_{G}(x) \\
& =\int_{G / H} \int_{H} \chi_{K}(\dot{x}+y) \chi_{\pi^{-1}(\tilde{E})}(\dot{x}+y) d m_{H}(y) d m_{G / H}(\dot{x}) \\
& =\int_{G / H} \chi_{\tilde{E}}(\dot{x}) \int_{H} \chi_{K}(\dot{x}+y) d m_{G}(y) d m_{G / H}(\dot{x}) \\
& =0 .
\end{aligned}
$$

Hence $\pi^{-1}(\tilde{E})$ is a locally null set and the proof is complete.

LeMMA 2.4. Let $G$ be a $\sigma$-compact metrizable LCA group and $P$ a closed semigroup in $\hat{G}$ such that $P \cup(-P)=\hat{G}$. Put $\Lambda=P \cap(-P)$ and $H=\Lambda^{\perp}$. Let $\pi$ be the natural homomorphism from $G$ onto $G / H$. For a measure $\mu \in M(G)$, we assume that there exists a family $\left\{\lambda_{\dot{x}}\right\}_{\dot{x} \in G / H}$ in $M(G)$ with the following properties:

(1) $\dot{x} \mapsto \lambda_{\dot{x}}(f)$ is a Borel measurable function for each bounded Borel function $f$ on $G$,

(2) $\operatorname{supp}\left(\lambda_{\dot{x}}\right) \subset \pi^{-1}(\{\dot{x}\})$, 
(3) $\left\|\lambda_{\dot{x}}\right\| \leq 1$,

(4) $\mu(g)=\int_{G / H} \lambda_{\dot{x}}(g) d \eta(\dot{x})$ for each bounded Borel measurable function $g$ on $G$,

where $\eta=\pi(|\mu|)$. Then the following is satisfied:

(5) If $\hat{\mu}(\gamma)=0$ on $P, \hat{\lambda}_{\dot{x}}(\gamma)=0$ on $P$ a.a. $\dot{x}(\eta)$.

Proof. First we note

$$
P+\Lambda \subset P \text {. }
$$

For $f \in L^{1}(\hat{G})$ with $\operatorname{supp}(f) \subset P$, we have

$$
\begin{aligned}
0 & =\int_{\hat{G}} \hat{\mu}(\gamma) f(\gamma) d \gamma \\
& =\int_{G} \hat{f}(x) d \mu(x) \\
& =\int_{G / H} \lambda_{\dot{x}}(\hat{f}) d \eta(\dot{x}) .
\end{aligned}
$$

On the other hand, for $\gamma_{*} \in \Lambda$, by (6), we have $\operatorname{supp}\left(f_{\gamma_{*}}\right) \subset P$, where $f_{\gamma_{*}}(\gamma)=f\left(\gamma-\gamma_{*}\right)$. Hence, by (7), we have

$$
\begin{aligned}
0 & =\int_{G / H} \lambda_{\dot{x}}\left(\hat{f}_{\gamma_{*}}\right) d \eta(\dot{x}) \\
& =\int_{G / H} \int_{G} \hat{f}_{\gamma_{*}}(x) d \lambda_{\dot{x}}(x) d \eta(\dot{x}) \\
& =\int_{G / H} \int_{G}\left(-x, \gamma_{*}\right) \hat{f}(x) d \lambda_{\dot{x}}(x) d \eta(\dot{x}) \\
& \left.=\int_{G / H}\left(-\dot{x}, \gamma_{*}\right) \int_{G} \hat{f}(x) d \lambda_{\dot{x}}(x) d \eta(\dot{x}) \quad \text { by }(2) \text { and } \gamma_{*} \in \Lambda\right) \\
& =\int_{G / H}\left(-\dot{x}, \gamma_{*}\right) \lambda_{\dot{x}}(\hat{f}) d \eta(\dot{x}) .
\end{aligned}
$$

Since $\gamma_{*}$ is an arbitrary element in $\Lambda$, we have

$$
0=\int_{G / H} p(\dot{x}) \lambda_{\dot{x}}(\hat{f}) d \eta(\dot{x}) \quad \text { for all } p(\dot{x}) \in \operatorname{Trig}(g / H) .
$$

Since $\operatorname{Trig}(G / H)$ is dense in $L^{1}(\eta)$ and $\dot{x} \mapsto \lambda_{\dot{x}}(\hat{f})$ is a bounded Borel measurable function, we have

$$
\lambda_{\dot{x}}(\hat{f})=0 \text { a.a. } \dot{x}(\eta) \text { for } f \in L^{1}(\hat{G}) \text { with } \operatorname{supp}(f) \subset P \text {. }
$$


Hence, for $f \in L^{1}(\hat{G})$ with $\operatorname{supp}(f) \subset P$, we have

$$
\begin{aligned}
0 & =\int_{G} \hat{f}(x) d \lambda_{\dot{x}}(x) \\
& =\int_{\hat{G}} \hat{\lambda}_{\dot{x}}(\gamma) f(\gamma) d \gamma \quad \text { a.a. } \dot{x}(\eta) .
\end{aligned}
$$

On the other hand, since $\hat{G}$ is $\sigma$-compact and metrizable, there exists a countable subset $\mathbb{Q}=\left\{f_{n}\right\}$ of $L^{1}(P)=\left\{f \in L^{1}(\hat{G}) ; \operatorname{supp}(f) \subset P\right\}$ such that it is dense in $L^{1}(P)$. By (10), for each $m \in N$ (the natural numbers), there exists a Borel set $\tilde{K}_{m}$ in $G / H$ such that $\eta\left(\tilde{K}_{m}^{c}\right)=0$ and

$$
0=\int_{\hat{G}} \hat{\lambda}_{\dot{x}}(\gamma) f_{m}(\gamma) d \gamma \quad \text { for } \dot{x} \in \tilde{K}_{m} .
$$

Put $K=\cap_{1}^{\infty} \tilde{K}_{m}$. Then $\eta\left(\tilde{K}^{c}\right)=0$ and

$$
0=\int_{\hat{G}} \hat{\lambda}_{\dot{x}}(\gamma) f_{m}(\gamma) d \gamma \quad \text { for all } \dot{x} \in \tilde{K} \text { and } f_{m} \in \mathscr{Q}
$$

Hence,

$$
0=\int_{\hat{G}} \hat{\lambda}_{\dot{x}}(\gamma) f(\gamma) d \gamma \quad \text { for all } \dot{x} \in \tilde{K} \text { and } f \in L^{1}(P),
$$

which yields

$$
\hat{\lambda}_{\dot{x}}(\gamma)=0 \quad \text { on } P \text { a.a. } \dot{x}(\eta)
$$

This completes the proof.

LEMMA 2.5. Let $\mathrm{G}$ be a $\sigma$-compact metrizable LCA group and $H$ a closed subgroup of $G$. Let $\pi$ be the natural homomorphism from $G$ onto $G / H$. Let $\left\{\lambda_{\dot{x}}\right\}_{\dot{x} \in G / H}$ be a family in $M^{+}(G)$ with the following properties:

(1) $\dot{x} \mapsto \lambda_{\dot{x}}(f)$ is a Borel measurable function for each bounded Borel measurable function $f$ on $G$,

(2) $\operatorname{supp}\left(\lambda_{\dot{x}}\right) \subset \pi^{-1}(\{\dot{x}\})$,

(3) $\left\|\lambda_{\dot{x}}\right\| \leq 1$.

By (2), $\lambda_{\dot{x}}=\nu_{\dot{x}} * \delta_{x}$ for some $\nu_{\dot{x}} \in M^{+}(H)$ and $x \in G$ with $\pi(x)=\dot{x}$. We define measures $\lambda_{\dot{x}}^{a}, \lambda_{\dot{x}}^{s} \in M^{+}(G)$ as follows:

(4) $\lambda_{\dot{x}}^{a}=\nu_{\dot{x}}^{a} * \delta_{x}, \lambda_{\dot{x}}^{s}=\nu_{\dot{x}}^{s} * \delta_{x}$, where $\nu_{\dot{x}}^{a}$ and $\nu_{\dot{x}}^{s}$ are the absolutely continuous part and the singular part of $\nu_{\dot{x}}$ with respect to $m_{H}$ respectively. Then the following is satisfied:

(5) $\dot{x} \mapsto \lambda_{\dot{x}}^{a}(f)$ and $\dot{x} \mapsto \lambda_{\dot{x}}^{s}(f)$ are Borel measurable functions for each bounded Borel function $f$ on $G$. 
Proof. For $\dot{x} \in G / H$, let $L^{1}\left(\pi^{-1}(\{\dot{x}\})\right)$ be the space of functions on $\pi^{-1}(\{\dot{x}\})$ which are integrable with respect to $m_{\dot{x}}$, where $m_{\dot{x}}$ is the measure on the coset $\pi^{-1}(\{\dot{x}\})$ which is given by translating $m_{H}$ on $\pi^{-1}(\{\dot{x}\})$.

Step 1. There exists a countable dense subset $\mathscr{Q}$ of $L^{1}(G)$ such that $\left.\mathbb{Q}\right|_{\pi^{-1}(\{\dot{x}\})}$ is dense in $L^{1}\left(\pi^{-1}(\{\dot{x}\})\right)$ for each $\dot{x} \in G / H$.

Since $G$ is $\sigma$-compact and metrizable, there exist open sets $U_{n}$ in $G$ with compact closures such that $\bar{U}_{n} \subset U_{n+1}$ and $\cup_{1}^{\infty} U_{n}=G$. Then, for each $n \in N$, there exists a countable set $\mathscr{Q}_{n}$ in $C_{c}(G)$ such that

(6) $\operatorname{supp}(f) \subset U_{n}$ for $f \in \mathbb{Q}_{n},\left.\mathbb{Q}_{n}\right|_{U_{n}}$ is dense in $C_{c}\left(U_{n}\right)$ with respect to the supremum norm.

Now we put $\mathbb{Q}=\cup_{1}^{\infty} \mathbb{Q}_{n}$. Then, by (6), $\mathscr{Q}$ is a countable dense subset of $L^{1}(G)$. Put $S_{n, \dot{x}}=\pi^{-1}(\{\dot{x}\}) \cap U_{n}$ and $B_{n, \dot{x}}=\left\{u \in C_{c}\left(\pi^{-1}(\{\dot{x}\})\right) ; \operatorname{supp}(u)\right.$ $\left.\subset S_{n, \dot{x}}\right\}$.

Claim 1. $\left.\mathbb{Q}_{n}\right|_{S_{n, \dot{x}}}$ is dense in $B_{n, \dot{x}}$. In fact, let $u$ be a function in $B_{n, \dot{x}}$ and $\varepsilon$ a positive real number. By Tietze's extension theorem, there exists a bounded continuous function $k_{n}$ on $G$ such that $\left.k_{n}\right|_{\bar{S}_{n, \dot{x}}}=\left.u\right|_{\bar{S}_{n, \dot{x}}}$ where $\bar{S}_{n, \dot{x}}$ is the closure of $S_{n, \dot{x}}$ in $\pi^{-1}(\{\dot{x}\})$. We choose an open set $V_{n}$ in $G$ and a nonnegative continuous function $p_{n}$ on $G$ with the compact support such that

$$
\begin{gathered}
\bar{V}_{n} \subset U_{n} \text { and } \operatorname{supp}(u) \subset V_{n}, \\
p_{n}= \begin{cases}1 & \text { for } x \in \bar{V}_{n}, \\
0 & \text { for } x \notin U_{n}\end{cases}
\end{gathered}
$$

and $\left\|p_{n}\right\|_{\infty} \leq 1$. Put $u_{n}(x)=k_{n}(x) p_{n}(x)$. Then $u_{n}$ is a continuous function on $G$ such that $\operatorname{supp}\left(u_{n}\right) \subset U_{n}$. Moreover, by the construction of $u_{n}$, we have $\left.u_{n}\right|_{s_{n, \dot{x}}}=\left.u\right|_{s_{n, \dot{x}}}$. Since $\left.\mathbb{Q}_{n}\right|_{U_{n}}$ is dense in $C_{c}\left(U_{n}\right)$, there exists a function $f_{n}$ in $\mathbb{Q}_{n}$ such that $\left\|\left.f_{n}\right|_{U_{n}}-\left.u_{n}\right|_{U_{n}}\right\|_{\infty}<\varepsilon$. Hence we have

$$
\begin{aligned}
\left\|\left.f_{n}\right|_{S_{n, \dot{x}}}-\left.u\right|_{S_{n, \dot{x}}}\right\|_{\infty} & =\left\|\left.f_{n}\right|_{S_{n, \dot{x}}}-\left.u_{n}\right|_{S_{n, \dot{x}}}\right\|_{\infty} \\
& \leq\left\|\left.f_{n}\right|_{U_{n}}-\left.u_{n}\right|_{U_{n}}\right\|_{\infty} \\
& <\varepsilon .
\end{aligned}
$$

Thus Claim is proved.

We return to the proof of Step 1. Let $f$ be a function in $L^{1}\left(\pi^{-1}(\{\dot{x}\})\right)$ and $\varepsilon$ a positive real number. Since $\cup_{1}^{\infty} S_{n, \dot{x}}=\pi^{-1}(\{\dot{x}\})$, there exists a positive integer $n$ such that $\int_{\left(S_{n, \dot{x}}\right)}|f(y)| d m_{\dot{x}}(y)<\varepsilon / 3$. We can also 
choose a function $f_{n} \in B_{n, \dot{x}}$ such that $\int_{S_{n, \dot{x}}}\left|f(y)-f_{n}(y)\right| d m_{\dot{x}}(y)<\varepsilon / 3$. By Claim 1, there exists a function $g_{n} \in \mathscr{Q}_{n}$ such that $\left\|\left.g_{n}\right|_{S_{n, \dot{x}}}-\left.f_{n}\right|_{S_{n, \dot{x}}}\right\|_{\infty}$ $<\varepsilon / 3\left(m_{\dot{x}}\left(S_{n, \dot{x}}\right)+1\right)$. Noting $\left.g_{n}\right|_{\pi^{-1}(\{\dot{x}\})}(y)=0$ if $y \in \pi^{-1}(\{x\}) \backslash S_{n, \dot{x}}$, we have

$$
\begin{aligned}
\int_{\pi^{-1}(\{\dot{x}\})}\left|f(y)-g_{n}(y)\right| d m_{\dot{x}}(y) \\
=\int_{\pi^{-1}(\{\dot{x}\}) \backslash S_{n, \dot{x}}}|f(y)| d m_{\dot{x}}(y)+\int_{S_{n, \dot{x}}}\left|f(y)-g_{n}(y)\right| d m_{\dot{x}}(y) \\
<\varepsilon / 3+\int_{S_{n, \dot{x}}}\left|f(y)-f_{n}(y)\right| d m_{\dot{x}}(y) \\
\quad+\int_{S_{n, \dot{x}}}\left|f_{n}(y)-g_{n}(y)\right| d m_{\dot{x}}(y) \\
<\varepsilon .
\end{aligned}
$$

Thus Step 1 is proved. In order to prove the lemma, it is sufficient to show that $\dot{x} \mapsto \lambda_{\dot{x}}^{s}(f)$ is a Borel measurable function for each $f \in C_{0}(G)$.

Step 2. For a nonnegative function $f \in C_{0}(G), \dot{x} \mapsto \lambda_{\dot{x}}^{s}(f)$ is a Borel measurable function.

Let $Q$ be the countable subset of $L^{1}(G)$ obtained in Step 1 and $\Re$ a countable dense subset of $C_{0}(G)$. Then we have

$$
\begin{aligned}
\lambda_{\dot{x}}^{s}(f) & =\left\|f \lambda_{\dot{x}}^{s}\right\| \\
& =\inf _{g \in \mathbb{Q}}\left\|f \lambda_{\dot{x}}-\chi_{\pi^{-1}(\{\dot{x}\})} g\right\| \\
& =\inf _{g \in \mathfrak{Q}} \sup _{\substack{h \in \mathscr{O}^{\prime} \\
\|h\|_{\infty} \leq 1}}\left|\lambda_{\dot{x}}(f h)-\left(\chi_{\pi^{-1}(\{\dot{x}\})} g\right)(h)\right| \\
& =\inf _{g \in \mathbb{Q}} \sup _{\substack{h \in \mathscr{B} \\
\|h\|_{\infty} \leq 1}}\left|\lambda_{\dot{x}}(f h)-\int_{\pi^{-1}(\{\dot{x}\})} g(z) h(z) d m_{\dot{x}}(z)\right| .
\end{aligned}
$$

We note that $\int_{\pi^{-1}(\{\dot{x}\})} g(z) h(z) d m_{\dot{x}}(z)=\int_{H} g(\dot{x}+y) h(\dot{x}+y) d m_{H}(y)$. Hence, $\dot{x} \mapsto \int_{\pi^{-1}(\{\dot{x}\})} g(z) h(z) d m_{\dot{x}}(z)$ is a continuous function on $G / H$. Therefore, by (1)and (8), Step 2 is proved.

By Step 2, $\dot{x} \mapsto \lambda_{\dot{x}}^{s}(f)$ is a Borel measurable function for each bounded Borel measurable function $f$ on $G$. This completes the proof. 
LEMMA 2.6. Let $G$ be a $\sigma$-compact metrizable LCA group and $P$ a closed semigroup in $\hat{G}$ such that $P \cup(-P)=\hat{G}$. Let $\mu$ be a measure in $M_{P^{c}}(G)$. Then $\mu_{a}$ and $\mu_{s}$ belong to $M_{P c}(G)$.

Proof. Put $\Lambda=P \cap(-P)$ and $H=\Lambda^{\perp}$. Let $\pi$ be the natural homomorphism from $G$ onto $G / H$, and put $\eta=\pi(|\mu|)$. Then, by Lemma 2.1, there exists a family $\left\{\xi_{\dot{x}}\right\}_{\dot{x} \in G / H}$ in $M^{+}(G)$ with the following properties:

(1) $\dot{x} \mapsto \xi_{\dot{x}}(f)$ is a Borel measurable function for each bounded Borel measurable function $f$ on $G$,

(2) $\operatorname{supp}\left(\xi_{\dot{x}}\right) \subset \pi^{-1}(\{\dot{x}\})$,

(3) $\left\|\xi_{\dot{x}}\right\| \leq 1$,

(4) $|\mu|(g)=\int_{G / H} \xi_{\dot{x}}(g) d \eta(\dot{x})$ for each bounded Borel measurable function $g$ on $G$.

Let $h$ be a unimodular Borel measurable function on $G$ such that $\mu=$ $h|\mu|$. By (2), there exists a measure $\nu_{\dot{x}} \in M^{+}(H)$ and $x \in G$ such that $\pi(x)=\dot{x}$ and $\xi_{\dot{x}}=\nu_{\dot{x}} * \delta_{x}$. Let $\nu_{\dot{x}}^{a}$ and $\nu_{\dot{x}}^{s}$ be the absolutely continuous part and the singular part of $\nu_{\dot{x}}$ with respect to $m_{H}$ respectively. We define measures $\xi_{\dot{x}}^{a}$ and $\xi_{\dot{x}}^{s}$ in $M^{+}(G)$ by $\xi_{\dot{x}}^{a}=\nu_{\dot{x}}^{a} * \delta_{x}$ and $\xi_{\dot{x}}^{s}=\nu_{\dot{x}}^{s} * \delta_{x}$. Put $\eta=\eta_{a}+\eta_{s}$, where $\eta_{a} \in L^{1}(G / H) \cap M^{+}(G / H)$ and $\eta_{s} \in M_{s}(G / H) \cap$ $M^{+}(G / H)$. Then, by Lemma 2.5 , we can define $\Phi_{a a}, \Phi_{s a}, \Phi_{s} \in M^{+}(G)$ as follows:

$$
\begin{aligned}
\Phi_{a a}(f) & =\int_{G / H} \xi_{\dot{x}}^{a}(f) d \eta_{a}(\dot{x}), \\
\Phi_{s a}(f) & =\int_{G / H} \xi_{\dot{x}}^{s}(f) d \eta_{a}(\dot{x}), \\
\Phi_{s}(f) & =\int_{G / H} \xi_{\dot{x}}(f) d \eta_{s}(\dot{x}) \quad \text { for } f \in C_{0}(G) .
\end{aligned}
$$

Claim 1. $\Phi_{s a} \in M_{s}(G) \cap M^{+}(G)$.

We define a measure $\zeta_{\dot{x}}^{s} \in M_{s}(G) \cap M^{+}(G)$ as follows:

$$
\zeta_{\dot{x}}^{s}= \begin{cases}\left(1 /\left\|\xi_{\dot{x}}^{s}\right\|\right) \xi_{\dot{x}}^{s} & \text { if }\left\|\xi_{\dot{x}}^{s}\right\| \neq 0 \\ 0 & \text { if }\left\|\xi_{\dot{x}}^{s}\right\|=0\end{cases}
$$

Then we have $\Phi_{s a}(f)=\int_{G / H} \zeta_{\dot{x}}^{s}(f)\left\|\xi_{\dot{x}}^{s}\right\| d \eta_{a}(\dot{x})$ for $f \in C_{0}(G)$. By Lemma 2.5 , we can define a measure $\eta_{a}^{\prime} \in L^{1}(G / H) \cap M^{+}(G / H)$ by $\eta_{a}^{\prime}(\tilde{E})=$ $\int_{\tilde{E}}\left\|\xi_{\dot{x}}^{s}\right\| d \eta_{a}(\dot{x})$ for a Borel set $\tilde{E}$ in $G / H$. Then we have $\pi\left(\Phi_{s a}\right)=\eta_{a}^{\prime}$. In 
fact, for $g \in C_{0}(G / H)$, we get

$$
\begin{aligned}
\pi\left(\Phi_{s a}\right)(g) & =\int_{G} g \circ \pi(x) d \Phi_{s a}(x) \\
& =\int_{G / H} \zeta_{\dot{x}}^{s}(g \circ \pi)\left\|\xi_{\dot{x}}^{s}\right\| d \eta_{a}(\dot{x}) \\
& =\int_{G / H} g(\dot{x})\left\|\xi_{\dot{x}}^{s}\right\| d \eta_{a}(\dot{x}) \\
& =\int_{G / H} g(\dot{x}) d \eta_{a}^{\prime}(\dot{x})
\end{aligned}
$$

Hence, for $\left\{\zeta_{\dot{x}}^{s}\right\}_{\dot{x} \in G / H}$ and $\eta_{a}^{\prime}$, we have

(6) $\pi\left(\Phi_{s a}\right)=\eta_{a}^{\prime}$,

(7) $\dot{x} \mapsto \zeta_{\dot{x}}^{s}(f)$ is a Borel measurable function for each bounded Borel function $f$ on $G$,

(8) $\operatorname{supp}\left(\zeta_{\dot{x}}^{s}\right) \subset \pi^{-1}(\{\dot{x}\})$,

(9) $\left\|\zeta_{\dot{x}}^{s}\right\| \leq 1$,

(10) $\Phi_{s a}(g)=\int_{G / H} \zeta_{\dot{x}}^{s}(g) d \eta_{a}^{\prime}(\dot{x})$ for each bounded Borel measurable function $g$ on $G$

and

(11) $\zeta_{\dot{x}}^{s} * \delta_{-x} \in M_{s}(H)$, where $x$ is an element in $G$ such that $\pi(x)=\dot{x}$. Hence, by (6)-(11) and Lemma 2.2, Claim 1 is proved.

Claim 2. $\Phi_{s} \in M_{s}(G) \cap M^{+}(G)$.

This is obtained from Lemma 2.3.

Claim 3. $\Phi_{a a} \in L^{1}(G)$.

Let $E$ be a Borel measurable set in $G$ such that $m_{G}(E)=0$. Then, since

$$
0=m_{G}(E)=\int_{G / H} \int_{H} \chi_{E}(\dot{x}+y) d m_{H}(y) d m_{G / H}(\dot{x}),
$$

there exists a Borel set $\tilde{F}$ in $G / H$ with $m_{G / H}(\tilde{F})=0$ such that $m_{\dot{x}}\left(E \cap \pi^{-1}(\{\dot{x}\})\right)=0$ if $\dot{x} \notin \tilde{F}$, where $m_{\dot{x}}$ is the measure on the coset $\pi^{-1}(\{\dot{x}\})$ translated $m_{H}$ on $\pi^{-1}(\{\dot{x}\})$. Then we have

$$
\begin{aligned}
\Phi_{a a}(E) & =\int_{G / H} \xi_{\dot{x}}^{a}\left(\chi_{E}\right) d \eta_{a}(\dot{x}) \\
& =\int_{\tilde{F}} \xi_{\dot{x}}^{a}\left(\chi_{E}\right) d \eta_{a}(\dot{x})+\int_{\tilde{F}^{c}} \xi_{\dot{x}}^{a}\left(\chi_{E}\right) d \eta_{a}(\dot{x}) . \\
& =0 .
\end{aligned}
$$

Thus Claim 3 is proved. 
We define a measure $\lambda_{\dot{x}} \in M(G)$ by $\lambda_{\dot{x}}(f)=\xi_{\dot{x}}(h f)$ for $f \in C_{0}(G)$, where $h$ is the unimodular Borel function on $G$ such that $\mu=h|\mu|$. Then the following are satisfied:

(12) $\dot{x} \mapsto \lambda_{\dot{x}}(f)$ is a Borel measurable function for each bounded Borel measurable function $f$ on $G$,

(13) $\operatorname{supp}\left(\lambda_{\dot{x}}\right)=\operatorname{supp}\left(\xi_{\dot{x}}\right) \subset \pi^{-1}(\{\dot{x}\})$,

(14) $\left\|\lambda_{\dot{x}}\right\| \leq 1$,

(15) $\mu(g)=\int_{G / H} \lambda_{\dot{x}}(g) d \eta(\dot{x})$ for each bounded Borel measurable function $g$ on $G$.

We define measures $\lambda_{\dot{x}}^{a}, \lambda_{\dot{x}}^{s} \in M(G)$ by $\lambda_{\dot{x}}^{a}=h \xi_{\dot{x}}^{a}$ and $\lambda_{\dot{x}}^{s}=h \xi_{\dot{x}}^{s}$ respectively. Then we have

$$
\lambda_{\dot{x}}=\lambda_{\dot{x}}^{a}+\lambda_{\dot{x}}^{s} \quad \text { for } \dot{x} \in G / H, \text { and }
$$

(16) $\lambda_{\dot{x}}^{a}$ and $\lambda_{\dot{x}}^{s}$ are absolutely continuous and singular with respect to $m_{\dot{x}}$ respectively.

By (13), there exist an element $x$ in $G$ with $\pi(x)=\dot{x}$ and a measure $\omega_{\dot{x}} \in M(H)$ such that $\lambda_{\dot{x}}=\omega_{\dot{x}} * \delta_{x}, \lambda_{\dot{x}}^{a}=\omega_{\dot{x}}^{a} * \delta_{x}$ and $\lambda_{\dot{x}}^{s}=\omega_{\dot{x}}^{s} * \delta_{x}$, where $\omega_{\dot{x}}^{a}$ and $\omega_{\dot{x}}^{s}$ are the absolutely continuous part and the singular part of $\omega_{\dot{x}}$ with respect to $m_{H}$ respectively. Since $\hat{\mu}(\gamma)=0$ on $P$, by Lemma 2.4 , we have

$$
\hat{\lambda}_{\dot{x}}(\gamma)=0 \text { on } P \text { a.a. } \dot{x}(\eta)
$$

hence

$$
\hat{\omega}_{\dot{x}}(\gamma)=0 \quad \text { on } P \text { a.a. } \dot{x}(\eta)
$$

Let $\beta$ be the natural homomorphism from $\hat{G}$ onto $\hat{G} / \Lambda$. Then $\beta(P)$ is a closed semigroup in $\hat{G} / \Lambda$. We note that $\beta(P)$ induces a totally order on $\hat{G} / \Lambda$, and moreover, $\beta(P)=\{\beta(\gamma) \in \hat{G} / \Lambda ; \beta(\gamma) \geq 0\}$. Hence, by (18) and Theorem $\mathrm{B}$, we have

$$
\hat{\omega_{\dot{x}}^{a}}(\gamma)=\hat{\omega_{\dot{x}}^{s}}(\gamma)=0 \quad \text { on } P \text { a.a. } \dot{x}(\eta)
$$

hence

$$
\hat{\lambda_{\dot{x}}^{a}}(\gamma)=\hat{s_{\dot{x}}}(\gamma)=0 \quad \text { on } P \text { a.a. } \dot{x}(\eta)
$$

On the other hand, by Lemma 2.5 and the construction of $\lambda_{\dot{x}}^{a}$ and $\lambda_{\dot{x}}^{s}$, $\dot{x} \mapsto \lambda_{\dot{x}}^{a}(f)$ and $\dot{x} \mapsto \lambda_{\dot{x}}^{s}(f)$ are Borel measurable functions for each bounded Borel measurable function $f$ on $G$. Hence we can define measures 
$\mu_{\imath} \in M(G)(i=1,2,3)$ as follows:

$$
\begin{aligned}
& \mu_{1}(f)=\int_{G / H} \lambda_{\dot{x}}^{a}(f) d \eta_{a}(\dot{x}), \\
& \mu_{2}(f)=\int_{G / H} \lambda_{\dot{x}}^{s}(f) d \eta_{a}(\dot{x}), \\
& \mu_{3}(f)=\int_{G / H} \lambda_{\dot{x}}(f) d \eta_{s}(\dot{x}) \quad \text { for } f \in C_{0}(G) .
\end{aligned}
$$

Then $\mu=\mu_{1}+\mu_{2}+\mu_{3}$, and, by the construction of $\lambda_{\dot{x}}, \lambda_{\dot{x}}^{a}$ and $\lambda_{\dot{x}}^{s}$, we have

$$
\mu_{1} \ll \Phi_{a a}, \quad \mu_{2} \ll \Phi_{s a} \text { and } \mu_{3} \ll \Phi_{s} .
$$

Therefore, by Claims 1-3, we have $\mu_{a}=\mu_{1}$ and $\mu_{s}=\mu_{2}+\mu_{3}$. By (20) and (21), we can easily verify that $\mu_{i} \in M_{P^{c}}(G)(i=1,2,3)$. Hence we have $\mu_{a}, \mu_{s} \in M_{P c}(G)$ and the proof is complete.

\section{Proof of Main Theorem.}

LEMMA 3.1. Let $G$ be a metrizable LCA group and $P$ a proper closed semigroup in $\hat{G}$ such that $P \cup(-P)=\hat{G}$. Let $\mu$ be a measure in $M(G)$. Then there exists a $\sigma$-compact open subgroup $G_{1}$ of $G$ such that $(1) \operatorname{supp}(\mu) \subset G_{1}$ and (2) $G_{1}^{\perp} \subset P \cap(-P)$.

Proof. Put $\Lambda=P \cap(-P)$, and let $\beta$ be the natural homomorphism from $\hat{G}$ onto $\hat{G} / \Lambda$. Then $\beta(P)$ is a closed semigroup in $\hat{G} / \Lambda$ such that (i) $\beta(P) \cup(-\beta(P))=\hat{G} / \Lambda$ and (ii) $\beta(P) \cap(-\beta(P))=\{0\}$. Hence, by ([8], 8.1.5. Theorem), we have

$$
\hat{G} / \Lambda=D, \text { or } \hat{G} / \Lambda=R \oplus D,
$$

where $D$ is a discrete abelian group which is torsion-free. Put $H=\Lambda^{\perp}$. Then, by (3), $H$ is a $\sigma$-compact closed subgroup of $G$. Since $\mu$ is regular, there exists a $\sigma$-compact open subgroup $G_{0}$ of $G$ such that $\operatorname{supp}(\mu) \subset G_{0}$. We put $G_{1}=G_{0}+H$. Then $G_{1}$ is a $\sigma$-compact open subgroup of $G$ which satisfies (1) and (2). This completes the proof.

LEMMA 3.2. Let $G$ be a metrizable LCA group and $P$ a closed semigroup in $\hat{G}$ such that $P \cup(-P)=\hat{G}$. Let $\mu$ be a measure in $M_{P c}(G)$. Then $\mu_{a}$ and $\mu_{s}$ belong to $M_{P^{c}}(G)$. 
Proof. We may assume that $P \subset \hat{G}$. Let $G_{1}$ be the $\sigma$-compact open subgroup of $G$ obtained in Lemma 3.1 . Let $\pi$ be the natural homomorphism from $\hat{G}$ onto $\hat{G} / G_{1}^{\perp}$. Then, by (2) in Lemma 3.1, $\pi(P)$ is a closed semigroup in $\hat{G} / G_{1}^{\perp}$ such that $\pi(P) \cup(-\pi(P))=\hat{G} / G_{1}^{\perp}$. We can regard $\mu$ as a measure in $M_{\pi(P) c}\left(G_{1}\right)$. Since $G_{1}$ is $\sigma$-compact and metrizable, by Lemma 2.6, we have $\mu_{a}, \mu_{s} \in M_{\pi(P)^{c}}\left(G_{1}\right)$, which yields $\mu_{a}, \mu_{s} \in M_{P^{c}}(G)$. This completes the proof.

Now we prove the main theorem of this paper.

TheOREM 3.3 (Main Theorem). Let $G$ be a LCA group and $P$ a closed semigroup in $\hat{G}$ such that $P \cup(-P)=\hat{G}$. Let $\mu$ be a measure in $M_{p^{c}}(G)$. Then $\mu_{a}$ and $\mu_{s}$ belong to $M_{P^{c}}(G)$.

Proof. It is sufficient to show that $\mu_{s} \in M_{P^{c}}(G)$. Let $\gamma_{0}$ be an element in $P$. Since $\mu_{s}$ is a singular measure, there exists a $\sigma$-compact subset $E$ of $G$ such that $m_{G}(E)=0$ and $\left|\mu_{s}\right|\left(E^{c}\right)=0$. Then, by ([7], Lemma 4), there exists a $\sigma$-compact open subgroup $\Gamma$ of $\hat{G}$ containing $\gamma_{0}$ such that

$$
m_{G}\left(E+\Gamma^{\perp}\right)=0 \text {. }
$$

Let $\pi$ be the natural homomorphism from $G$ onto $G / \Gamma^{\perp}$. Then, by (1), we have

$$
\pi(\mu)_{s}=\pi\left(\mu_{s}\right)
$$

Put $P_{\Gamma}=P \cap \Gamma$. Then $P_{\Gamma}$ is a closed semigroup in $\Gamma$ such that $P_{\Gamma} \cup$ $\left(-P_{\Gamma}\right)=\Gamma$, and $\pi(\mu)$ belongs to $M_{P_{\Gamma}^{\mathrm{c}}}\left(G / \Gamma^{\perp}\right)$. Since $G / \Gamma^{\perp}$ is metrizable, by (2) and Lemma 3.2, we have $\pi\left(\mu_{s}\right)=\pi(\mu)_{s} \in M_{P_{\Gamma}^{c}}\left(G / \Gamma^{\perp}\right)$, so that $\hat{\mu}_{s}\left(\gamma_{0}\right)=\pi\left(\mu_{s}\right) \hat{(}\left(\gamma_{0}\right)=0$. Since $\gamma_{0}$ is an arbitrary element in $P$, we have $\mu_{s} \in M_{P^{c}}(G)$. This completes the proof.

REMARK 3.4. In the proof of Lemma 2.6, when $\hat{G} / \Lambda$ is not discrete, we needed Theorem $\mathrm{B}$. However, in this case, we have $\hat{G} / \Lambda \cong R \oplus D$ and $\beta(P) \cong\{(x, d) \in R \oplus D ; d>0$, or $d=0$ and $x \geq 0\}$, where $D$ is a discrete ordered group (cf. [8], 8.1.5. Theorem). Using Theorem A and our method, we can prove Theorem B if $P$ is closed. Hence the Main Theorem can be obtained by employing only Theorem A. 
Appendix. The author has recently extended Theorem A(II) as follows (cf. [10], Lemma 1.2):

THEOREM 3.5. Let $G$ be a LCA group and $P$ a semigroup in $\hat{G}$ such that $P \cup(-P)=G$. Put $\Lambda=P \cap(-P)$ and $H=\Lambda^{\perp}$. If $P$ is open, then we have

$$
m_{H} *\left\{M_{P}(G) \cap M_{s}(G)\right\} \subset M_{P}(G) \cap M_{s}(G)
$$

\section{REFERENCES}

[1] N. Bourbaki, Intégration, Éléments de Mathématique, Livre VI, Ch. 6, Paris, Herman, 1959.

[2] K. deLeeuw and I. Glicksberg, Quasi-invariance and analyticity of measures on compact groups, Acta Math., 109 (1963), 179-205.

[3] R. Doss, On the Fourier-Stieltjes transforms of singular or absolutely continuous measures, Math. Z., 97 (1967), 77-84.

[4] _ On measures with small transforms, Pacific J. Math., 26 (1968), 257-263.

[5] I. Glicksberg, Fourier-Stieltjes transforms with small supports, Illinois. J. Math., 9 (1965), 418-427.

[6] H. Helson and D. Lowdenslager, Prediction theory and Fourier series in several variables, Acta Math., 99 (1958), 165-202.

[7] L. Pigno and S. Saeki, Fourier-Stieltjes transforms which vanish at infinity, Math. Z., 141 (1975), 83-91.

[8] W. Rudin, Fourier Analysis On Groups, New York, Interscience, 1962.

[9] H. Yamaguchi, On the product of a Riesz set and a small p set, Proc. Amer. Math. Soc., 81 (1981), 273-278.

[10] - Some multipliers on the space consisting of measures of analytic type, II, (to appear in Hokkaido. Math. J.).

Received March 9, 1981 and in revised form May 10, 1982.

JOSAI UNIVERSITY,

SaKado, SaItama, JAPAN 


\section{PACIFIC JOURNAL OF MATHEMATICS EDITORS}

Donald BABBITT (Managing Editor)

University of California

Los Angeles, CA 90024

Hugo Rossi

University of Utah

Salt Lake City, UT 84112

C. C. Moore and Arthur Ogus

University of California

Berkeley, CA 94720
J. DugundiI

Department of Mathematics

University of Southern California

Los Angeles, CA 90089-1113

R. Finn and H. SAMELSON

Stanford University

Stanford, CA 94305

ASSOCIATE EDITORS
R. ARENS
E. F. BECKENBACH
B. H. NeUmanN
F. WOLF
K. YosHIDA (1906-1982)

\section{SUPPORTING INSTITUTIONS}

UNIVERSITY OF ARIZONA

UNIVERSITY OF BRITISH COLUMBIA

CALIFORNIA INSTITUTE OF TECHNOLOGY

UNIVERSITY OF CALIFORNIA

MONTANA STATE UNIVERSITY

UNIVERSITY OF NEVADA, RENO

NEW MEXICO STATE UNIVERSITY

OREGON STATE UNIVERSITY
UNIVERSITY OF OREGON

UNIVERSITY OF SOUTHERN CALIFORNIA

STANFORD UNIVERSITY

UNIVERSITY OF HAWAII

UNIVERSITY OF TOKYO

UNIVERSITY OF UTAH

WASHINGTON STATE UNIVERSITY

UNIVERSITY OF WASHINGTON 


\section{Pacific Journal of Mathematics}

\section{Vol. 108, No. $1 \quad$ March, 1983}

Waleed A. Al-Salam and A. Verma, $q$-Konhauser polynomials $\ldots \ldots \ldots \ldots 1$

Alfred David Andrew, The Banach space JT is primary $\ldots \ldots \ldots \ldots \ldots . . .6$

Thomas E. Bengtson, Bessel functions on $P_{n} \ldots \ldots \ldots \ldots \ldots \ldots$

Joaquim Bruna Floris and Francesc Tugores, Free interpolation for

holomorphic functions regular to the boundary $\ldots \ldots \ldots \ldots \ldots \ldots \ldots \ldots$

Peter Dierolf and Susanne Dierolf, Topological properties of the dual pair

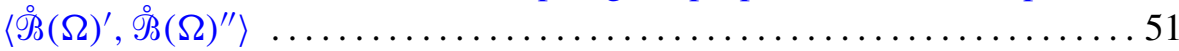

Gerald Arthur Edgar, An ordering for the Banach spaces $\ldots \ldots \ldots \ldots \ldots 83$

Basil Gordon, A proof of the Bender-Knuth conjecture . . . . . . . . . . . . . 99

Harold T. Hodes, A minimal upper bound on a sequence of Turing degrees

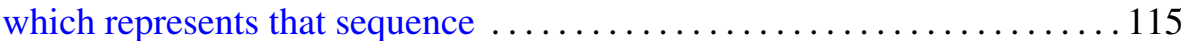

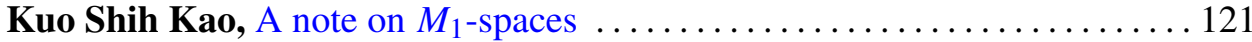

Frank Kost, Topological extensions of product spaces ................ 129

Eva Lowen-Colebunders, On the convergence of closed and compact

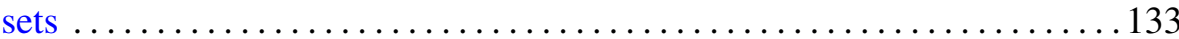

Doron Lubinsky, Divergence of complex rational approximations . . . . . . 141

Warren May and Elias Hanna Toubassi, Endomorphisms of rank one

mixed modules over discrete valuation rings $\ldots \ldots \ldots \ldots \ldots \ldots \ldots \ldots \ldots$

Richard Patrick Morton, The quadratic number fields with cyclic

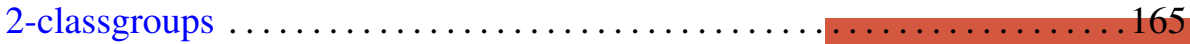

Roderic Murufas, Rank of positive matrix measures . . . . . . . . . . . . 177

Helga Schirmer, Fixed point sets of homotopies . . . . . . . . . . . . . 191

E. Taflin, Analytic linearization of the Korteweg-de Vries equation ........ 203

James Thomas Vance, Jr., $L^{p}$-boundedness of the multiple Hilbert

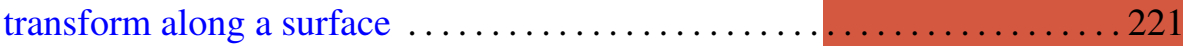

Hiroshi Yamaguchi, A property of some Fourier-Stieltjes transforms . . . . . 243 